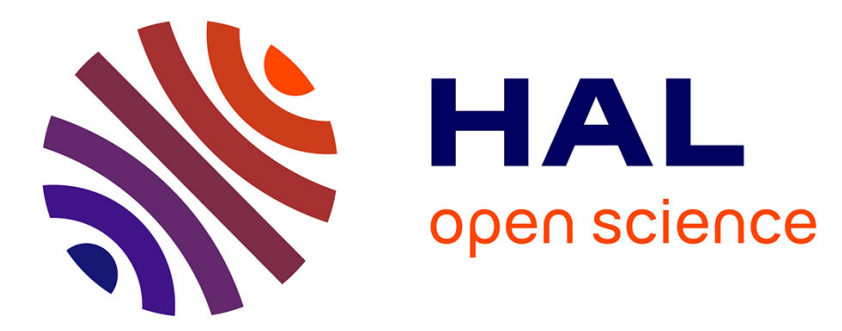

\title{
Multiphoton microscopy for pre-clinical evaluation of flow-diverter stents for treating aneurysms
}

Sylvia M. Bardet, Jonathan Cortese, Raphaël Blanc, Charbel Mounayer, Aymeric Rouchaud

\section{- To cite this version:}

Sylvia M. Bardet, Jonathan Cortese, Raphaël Blanc, Charbel Mounayer, Aymeric Rouchaud. Multiphoton microscopy for pre-clinical evaluation of flow-diverter stents for treating aneurysms. Journal de Neuroradiologie / Journal of Neuroradiology, 2021, 10.1016/j.neurad.2020.03.005 . hal-03010851

\section{HAL Id: hal-03010851 \\ https://hal.science/hal-03010851}

Submitted on 17 Nov 2020

HAL is a multi-disciplinary open access archive for the deposit and dissemination of scientific research documents, whether they are published or not. The documents may come from teaching and research institutions in France or abroad, or from public or private research centers.
L'archive ouverte pluridisciplinaire $\mathbf{H A L}$, est destinée au dépôt et à la diffusion de documents scientifiques de niveau recherche, publiés ou non, émanant des établissements d'enseignement et de recherche français ou étrangers, des laboratoires publics ou privés. 
Original article

\title{
Multiphoton microscopy for pre-clinical evaluation of flow-diverter stents for treating aneurysms
}

\author{
Sylvia M. Bardet ${ }^{\mathrm{a}, *}$, Jonathan Cortese ${ }^{\mathrm{b}, \mathrm{c}}$, Raphaël Blanc ${ }^{\mathrm{d}}$, Charbel Mounayer ${ }^{\mathrm{a}, \mathrm{e}}$, Aymeric Rouchaud ${ }^{\mathrm{a}, *, \mathrm{e}}$ \\ a University of Limoges, 123, avenue Albert-Thomas, XLIM UMR CNRS 7252, 87060 Limoges, France \\ b Bichat University Hospital, INSERM U1148-LVTS, Paris, France \\ ${ }^{\mathrm{c}}$ Bicetre Hospital, Department of Interventional Neuroradiology, Paris, France \\ d Department of Interventional Neuroradiology, Fondation Ophtalmologique Adolphe-de-Rothschild, Paris, France \\ e University Hospital, Department of Interventional Neuroradiology, Limoges, France
}

\section{A R T I C L E IN F O}

\section{Article history:}

Available online $\mathrm{xxx}$

Keywords:

Aneurysms

Endovascular treatment

Stenting

Flow-Diverter

Multiphoton microscopy

Second harmonic generation

\section{A B S T R A C T}

\section{Background}

Conventional histological analyses are the gold standard for the study of aneurysms and vascular pathologies in pre-clinical research. Over the past decade, in vivo and ex vivo imaging using multiphoton microscopy have emerged as powerful pre-clinical tools for detailed tissue analyses that can assess morphology, the extracellular matrix (ECM), cell density and vascularisation. Multiphoton microscopy allows for deeper tissue penetration with minor phototoxicity. Objective

The present study aimed to demonstrate the current status of multimodality imaging, including multiphoton microscopy, for detailed analyses of neo-endothelialisation and ECM evolution after flow-diverter stent (FDS) treatment in an experimental rabbit model of aneurysms.

Methods

Multiphoton microscopy tools for assessing autofluorescence and second harmonic generation (SHG) signals from biological tissues were used to evaluate the endovascular treatment of intracranial aneurysms in an animal model of aneurysms (pig, rabbit). Results from multiphoton microscopy were compared to those from standard histology, electronic and bright field microscopy. Conclusions

The present study describes novel evaluation modes based on multiphoton microscopy for visualising tissue morphology (e.g., collagen, elastin, and cells) to qualify and quantify the extent of neo-intimal formation of covered arteries and device integration into the arterial wall using a rabbit model of intracranial aneurysms treated with FDS.

(C) 2020

\section{Background}

Endovascular therapy is the gold standard treatment for intracranial aneurysms and has supplanted surgical approaches for most of aneurysms [1]. Flow-diverter stents (FDS) are widely accepted as a standard of care for the treatment of selected aneurysms [2-4]. Because the mechanisms underlying aneurysm occlusion following FDS treatment are likely related to stent endothelialisation, it is important to promote better endothelialisation at the neck of the aneurysm over time. Indeed, to better understand and assess the healing processes in-

\footnotetext{
Abbreviations: FD, Flow-Diverter; HBSS, Hank's Balanced Salt Solution; PMT, Photomultiplier tube; SHG, Second Harmonic Generation

* Corresponding authors.

Email addresses: sylvia.bardetcoste@unilim.fr (S.M. Bardet); aymeric.
} rouchaud@unilim.fr (A. Rouchaud) volved in aneurysm occlusion, it is necessary to develop performant tools that measure appropriate healing.

Over the last decade, several research groups have provided technical guides for in vivo imaging of vascular compartments using multiphoton microscopy of several organs [5-7]. Moreover, recent advances in photonic tools have provided novel results relative to classical histological approaches for assessing tissue architecture due to the ability to image deeper areas and render three-dimensional (3D) views of tissue. For example, two-photon excitation microscopy (TPEM) has emerged as an efficient tool for revealing the composition of cells and the extracellular matrix (ECM) based on elastin autofluorescence and second harmonic generation (SHG) signals from collagen [8]. This technique facilitates the imaging of living or fixed cells and tissues without the need for intravital labelling using chemical or genetic fluorescent dyes.

This type of non-linear microscopy is useful for healthy tissues as well as pathological models [9] of the elastic lamina of the arterial wall without the use of exogenous stains [10-13]. Recently, 

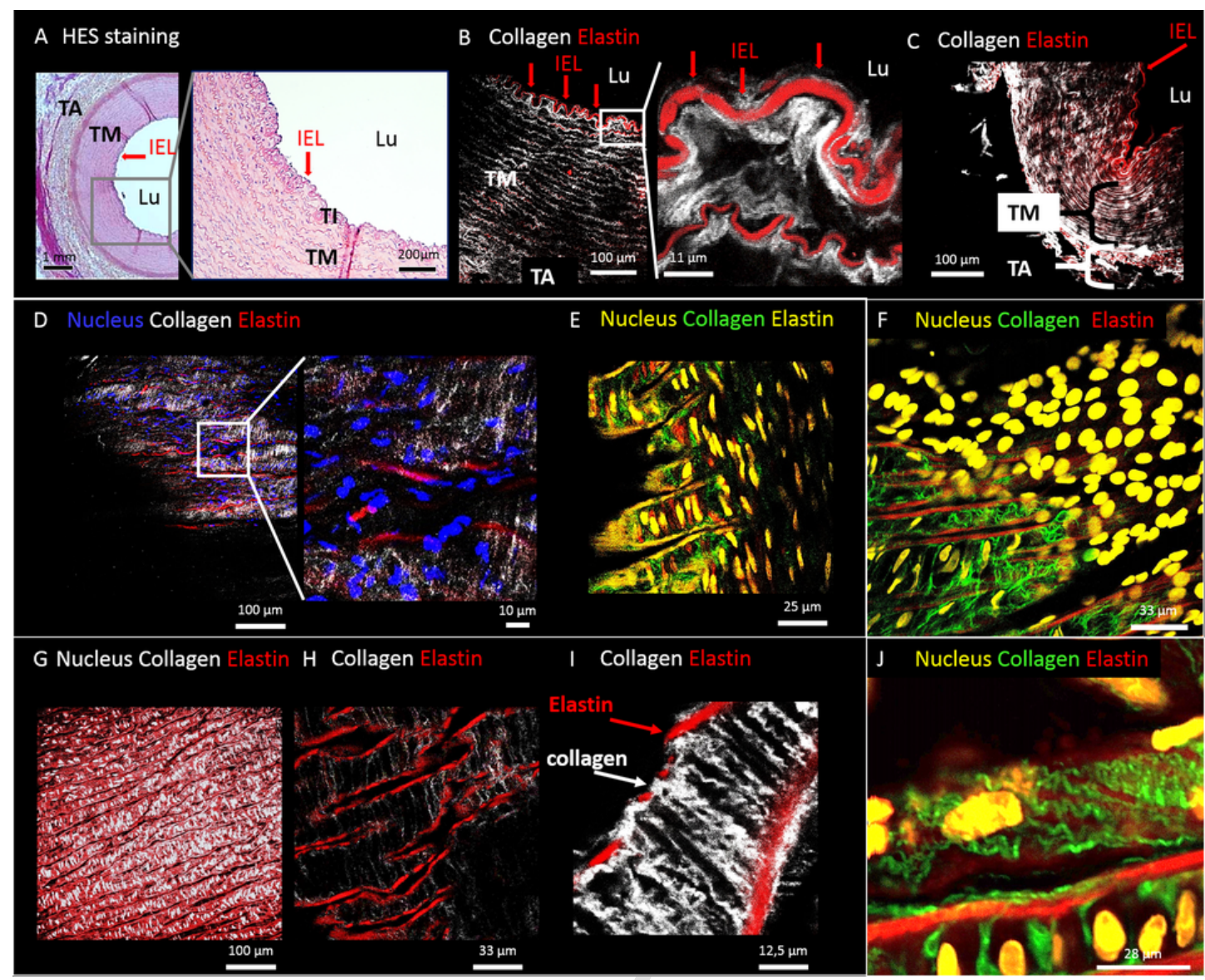

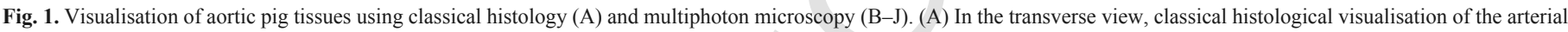

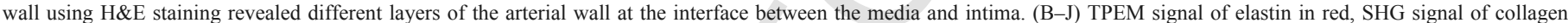

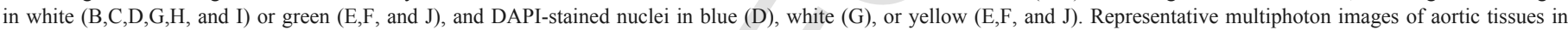

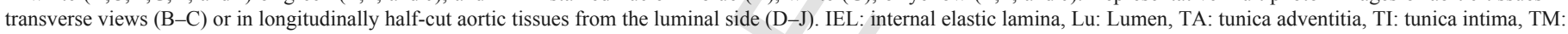
tunica media.

novel TPEM findings regarding the architectures of collagen and elastin fibres have provided information about the compartmentalisation between the luminal and abluminal sides of human cerebral aneurysms [14].

The present study focused on the importance of multimodal evaluations using optimised tools for the assessment of endothelialisation and ECM production. More specifically, the biointegration capabilities of FDS were evaluated using an ex vivo multiphoton technique in a model of aneurysms in rabbit and pig.

\section{Material and methods}

Animals and ethics statement

All animal care and experimental procedures were conducted in accordance with the 2013 French legislation and European Community guidelines (directive 2010/63/UE) and approved by the French National Ministry of Research (Optimisationdesstent_2018022812464127_v3.apafis\#5747).

\section{Pig arterial conventional histology}

Formol-fixed pig subrenal aortae were dehydrated and embedded in paraffin. 5- $\mu \mathrm{m}$ transverse sections were stained with hematoxylin and eosin to visualize the nuclei in blue/purple, cytoplasm and mus- cle cells in red, and collagen in pale pink. Unstained paraffin sections and aortae pieces were imaged using multiphoton microscopy for the visualization of collagen and elastin. Additionally, the entire aorta was cut in half longitudinally and then mounted with the tunica adventitia facing onto the glass slide for en face imaging. This allowed imaging with or without previous DAPI staining (Sigma-Aldrich).

\section{Rabbit aneurysms analyses}

Experimental aneurysms in rabbits using the elastase-induced model were created according to previously described technique [15]. Three weeks after, aneurysms were treated with FDS covering the ostium of the aneurysms [16,17]. The FDS used were Silk Vista Baby ${ }^{\circledR}$ FDS (Balt, France). One month after FDS implantation, the aneurysms and parent arteries were harvested for ex vivo analyses to evaluate endothelial cell colonisation. Scanning electron microscopy and multiphoton microscopy were performed on ex vivo explants of the stented arteries in the subrenal aorta.

\section{Tissue processing for microscopic imaging}

\section{Scanning electron microscope (SEM)}

The samples were disposed on appropriated aluminum stubs to observe the luminal side with the surface of the wall-apposed stent. Images were obtained using a gaseous secondary electron detec- 


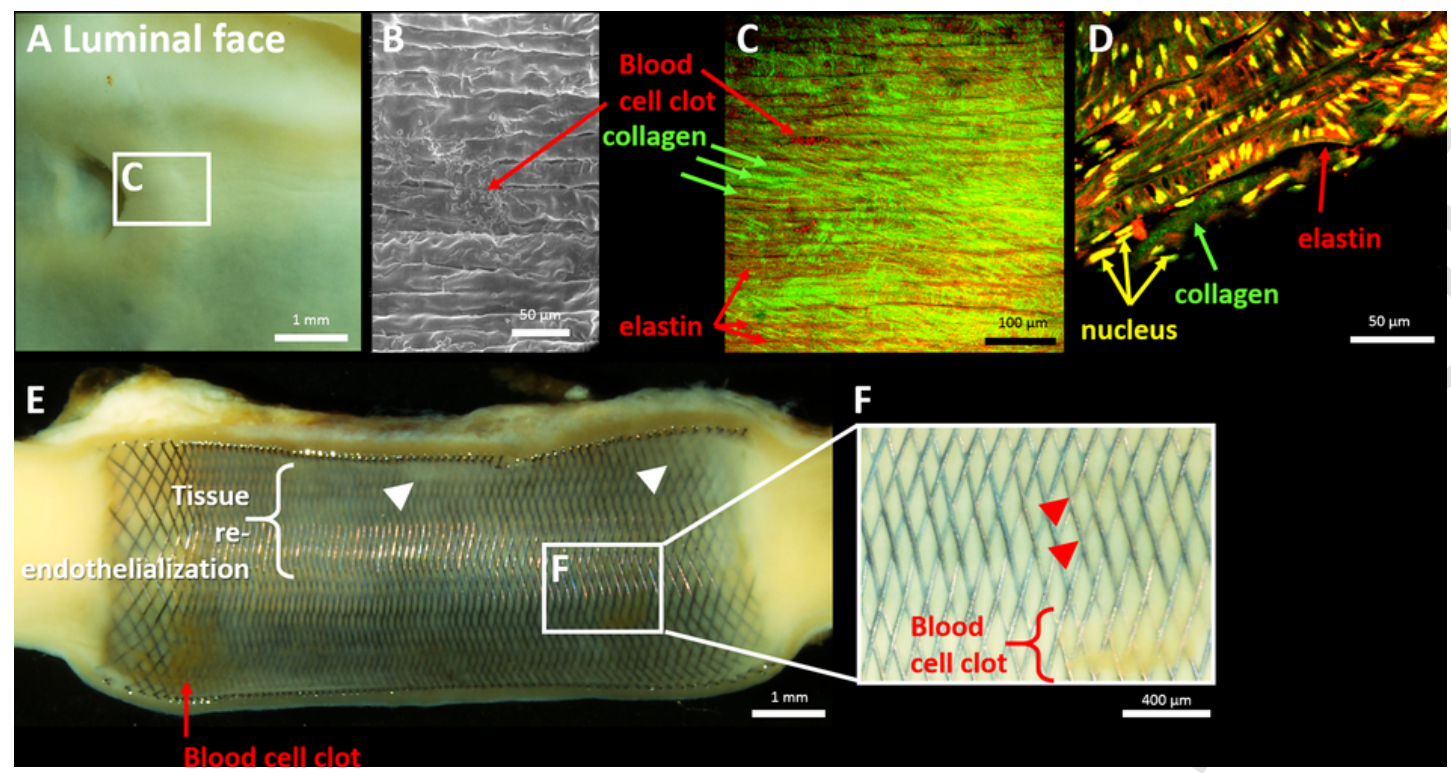

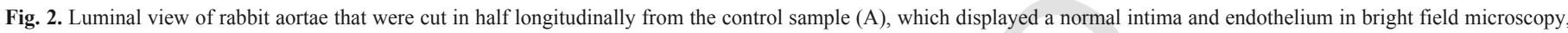

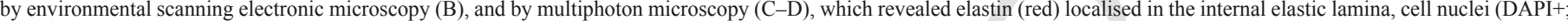

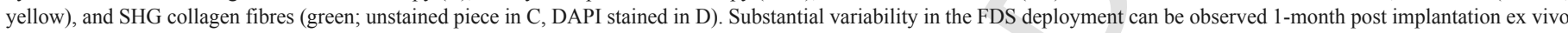
on the bright field stereomicroscopy images as the stent was deployed irregularly, much like an accordion with different mesh density areas (E-F).

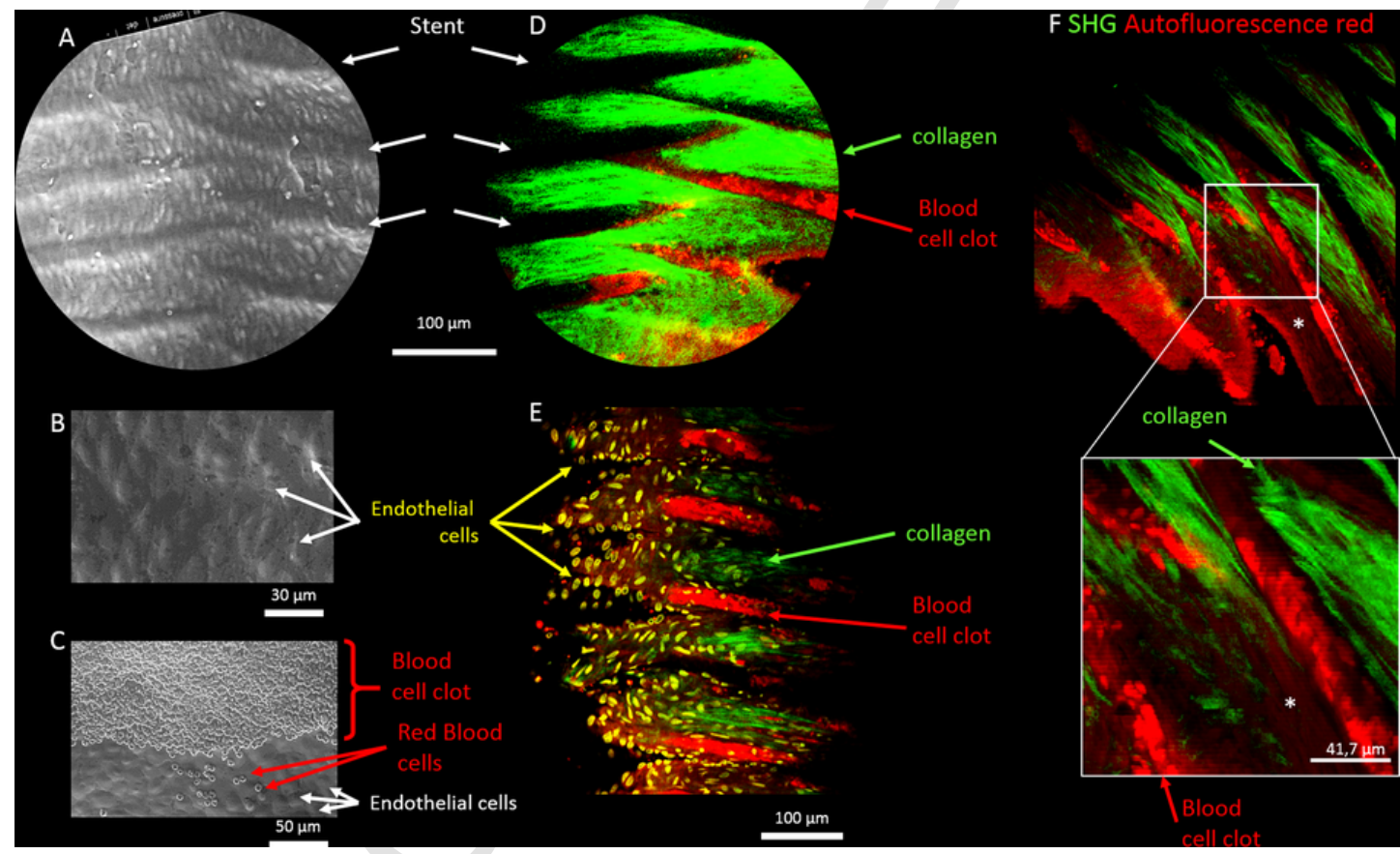

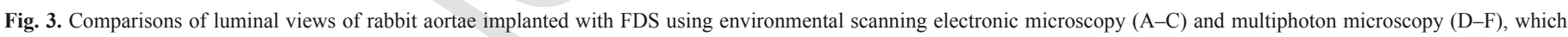

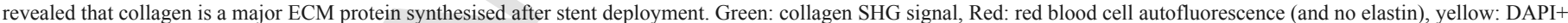
cells.

tor with a FEI-Quanta-450-FEG microscope at accelerating voltages of $7.5-10 \mathrm{kV}$, a spot size of 4 or 4.5 , and working distances of $10 \mathrm{~mm}$ and $7 \mathrm{~mm}$ in low vacuum and wet mode, respectively.

\section{Bright field microscopy}

The samples were fixed with pins in a dish and then covered with Live Cell Imaging Solution (Molecular Probes $\left.{ }^{\circledR}\right)$. A MVX10 Macro Zoom Microscope and a 17.28-megapixel cooled digital DP73 colour camera were used with CellSens Dimension imaging software (Olympus).

\section{Multiphoton microscopy}

Multiphoton microscopy allows for increased penetration depth into biological tissue with a WD of $400 \mu \mathrm{m}$ that yields high-resolution 3D images of the neo-intimal wall. The nuclei of the samples (rabbit and pig) were stained with DAPI (Sigma-Aldrich) in Live 

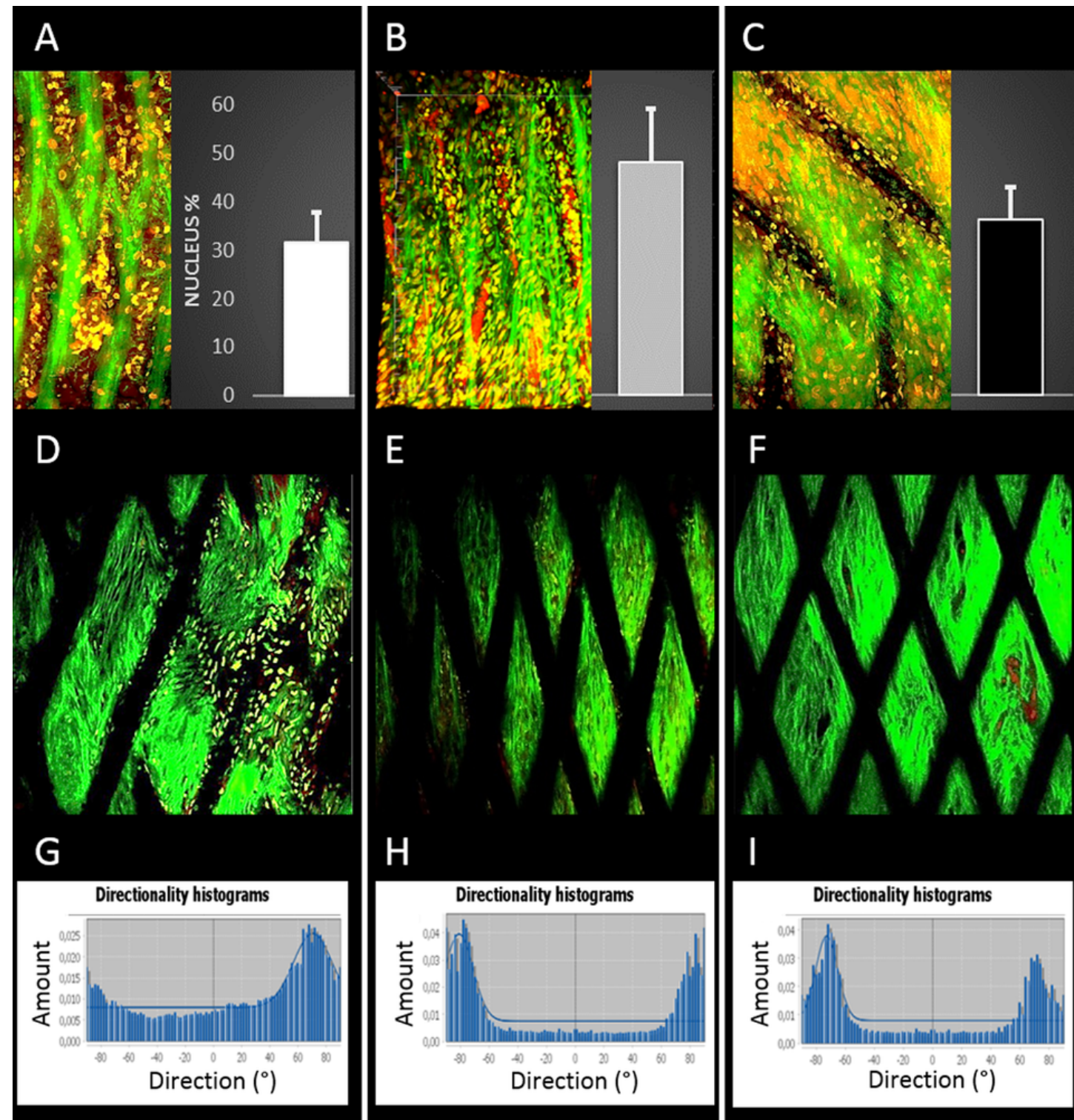

Fig. 4. Quantification of DAPI-positive cells (A-C) and SHG collagen signal directionality (D-I) in multiphoton images from three examples of coated metal stents that exhibited different reendothelialisation and ECM profile (type 1: A, D, and G; type 2: B, E, and $\mathrm{H}$; and type $3: \mathrm{C}, \mathrm{F}$, and I). $n=11,31.47 \% \pm 8.34 \mathrm{SD}$ in $\mathrm{A}, n=14 ; 47.9 \% \pm 15 \mathrm{SD}$ in $\mathrm{B}, n=11$; $36,09 \% \pm 9,32 \mathrm{SD}$ in C ( $n$ value corresponds to a mean of data obtained from different zones in each stent in several animals). Scale bar in E applied to all.

Cell Imaging Solution (Molecular Probes $\left.{ }^{\circledR}\right)$ for 5 min and then washed twice. A customised multiphoton microscope (BX61WI/ FV1200MPE, Olympus) was coupled with a linear polarisation femtosecond Ti:sapphire pulsed laser (Chameleon Ultra II, Coherent) (7). 3D multichannel stacks $(500 \times 500 \mu \mathrm{m})$ were collected at $2-\mu \mathrm{m}$ intervals with $512 \times 512$ pixels and a $12.5-\mu \mathrm{s} /$ pixel dwell time at $810 \mathrm{~nm}$ using FluoView FV1200 software (Olympus). Then, the different components of the light emitted from the sample were separated using a dichroic mirror $(570 \mathrm{~nm})$ that distributed the SHG signals and red fluorescence to two photomultiplier tubes (PMT) with fluorophore-specific emission filters (400-410 for SHG: green; 575-630 for autofluorescence: red; and both PMT for DAPI: yellow).

\section{Quantification and orientation of collagen fibres}

All images were background-corrected and median-filtered equally across samples after conversion to red/green/blue (RGB) or grayscale images. The images were segmented, rendered to $3 \mathrm{D}$ vol- umes, and measured with Imaris software (Bitplane AG). Collagen fibre orientation was calculated using the OrientationJ plugin (ImageJ, $\mathrm{NIH}$ ) [18]. Collagen quantification was performed after z-projection of the 3D raw images, splitting channels (RGB) to allow only green colour images to be kept, and establishing threshold ranges to better distinguish collagen fibres from the rest of the sample (artifacts or debris) and the image background. Collagen area (\%) was quantified using the "Analyse Particles" command [19].

\section{Quantification of endothelial cells}

The images were background-corrected and median-filtered equally across samples, segmented, and z-projected from 3D volumes using Image J (NIH) software. To specifically select the signals corresponding to DAPI staining on both PMT (red and green=yellow pseudocolors), a yellow colour extraction was done with Adobe Photoshop CS6, and the images were rendered to 8-bit grayscale (binary contrast enhancement). For each image, a threshold intensity proce- 
dure was carried out so that the fluorescent nuclei would appear as black objects on a white background. Then, particles were analysed to assess the circular areas of the pixels, and the cell nuclei were quantified using automated counting performed with ImageJ software [20] and estimation of the surface occupied by the nuclei were plotted on a graph with the mean in \% (error bars represent the standard deviation SD).

\section{Results}

Multiphoton en face imaging of the aorta allows for the acquisition of high-quality 3D images that can provide detailed assessments of microstructural modifications in the vascular wall. As a control (no stent), an entire pig aorta was encased in a paraffin block, and thin sections of this block were stained with $\mathrm{H} \& \mathrm{E}$ for histological analyses (Fig. 1A), without staining for TPEM (Fig. 1B-C and H-I), or with the previous DAPI stain for labelling cell nuclei (Fig. 1D-G and J). These processes permit the simultaneous visualisation of elastic lamellae via elastin autofluorescence by TPEM, collagen fibres by SHG, and cell nuclei by DAPI-TPEM. In this set of cross sections, it was possible to image the internal elastic lamellae (IEL; red) and the adventitial and medial collagen fibres (TA and TM, respectively, white; Fig. 1B-C, Supp. Video 1). In Fig. 1C, the outermost layer of the aorta is distinguishable from the media by denser bundles of collagen (TA vs. TM).

Other acquisitions consisted of en face z-stack imaging along the $\mathrm{X}-\mathrm{Y}$ axes (Fig. 1D-J) starting at the IEL and continuing to the TA. This process resulted in a normal loss of signal with depth due to the absorption and scattering of light because the power of the laser was not increased with the depth; this issue became evident at approximately $100 \mu \mathrm{m}$ inside the tissue. Fig. $1 \mathrm{G}$ provides an entire volumetric view of the aortic tunica; the intima layer, which is close to the lumen and is composed of a layer of endothelial cells (DAPI-positive cells; white), is attached to the elastin autofluorescence (IEL; red). This image shows many parallel and concentric layers of elastic fibres (red) that compose the medial layer as well as orthogonal nuclei from endothelial cells (white). These nuclei are clearly well organised in structured endothelial layers (Fig. 1D-F; nuclei in blue or yellow) that alternate with collagen and elastin (Supp. Video 2). Interestingly, it is possible to see interlaced elastin (red) wrapped with collagen (white) similar to boat ropes in Fig. 1I (arrows). In Fig. 1J, yellow nuclei are finely intercalated with smaller interconnecting wavy collagen fibres (green) and larger straight elastin (red).

To further explore reendothelialisation mechanisms after FDS implantation, multimodal microscopy was used to image the abluminal side of the rabbit aorta. In Fig. 2A-D, a control vascular wall (no stent) is shown in images from white-field microscopy (A) compared to electronic (B) and multiphoton (C, D) microscopy. The rabbit aortae display the same anatomy and ultrastructure as previously described in the pig aorta; i.e., elastin autofluorescence (C-D; red), collagen SHG signal (C-D; green) and DAPI-positive endothelial cells (D; yellow). In particular, uptake of glutaraldehyde by erythrocytes and molecule crosslinking inside increases autofluorescence drastically (see blood cell clot in Fig. 2B and red marks in Fig. 2C). Moreover, blood cells that adhere to tissues due to rapid coagulation during the biopsy can generate undesirable signals in the red channel (see next section).

Bright field stereomicroscopy revealed that the stent deployment allowed for coverage of the entire endothelial surface (Fig. 2E-F); nevertheless, the stent expanded irregularly, much like an accordion. Unfortunately, this phenomenon is challenging and laborious because of the delicate in vivo surgical procedure necessary to insert the FDS into the aorta. It is possible that the discrepancy in stent deployment might have influenced reendothelialisation because the endothe- lium coverage of the metallic mesh can vary from low density (Fig. 2E, left) to high density (zone F in Fig. 2E). Additionally, wall apposition can influence cell proliferation over the device [16]. In fact, the stent in Fig. 2E was covered by a white semi-transparent biological tissue (white arrows and white accolade pinpointing tissue reendothelialisation). Blood cell clots were also clearly visible as a reddish-orange colour (red arrows and red accolade); these could not be eliminated with subsequent washings due to the powerful fixation process that was applied to the aorta samples.

Next, the biological tissue that had synthesised on the surface of the metallic stents after the healing process ( 1 month) was characterised. To accomplish this, microscopy techniques with resolution higher than that of light microscopy were used (Fig. 3). Environmental scanning electron microscopy enables the investigation of hydrated and uncoated biological samples without degradation of the material. It was possible to obtain a detailed description of the structure and surface of the stent using a backscattered electron detector and a relevant material contrast (Fig. 3A-C). In Fig. 3A, the relief of the stent can be estimated but is not directly visible due to the presence of biological tissues composed of endothelial cells (Fig. 3B). In another location, red blood cells can be easily discriminated from the endothelial cells near a blood clot (Fig. 3C).

Of the various imaging modalities, multiphoton microscopy provides anatomical landmarks that are very similar to those observed using electronic microscopy but with greater detail and precision. In Fig. 3 D-E, the similarities are observable due to red autofluorescence, SHG signals, and fluorescent DAPI labelling. The stent appears black because it does not emit two-photon signals due to its metallic properties, whereas layers of collagen fibres (SHG; green) can be observed in the luminal endothelial cell zone (DAPI+; yellow) staggered with deeper blood cell clot layers that frequently adhered to the metallic surface of the stent. Unfortunately, these blood cells exhibit excessive autofluorescence in the red spectrum and can overlap with the expected elastin autofluorescence. Nevertheless, after stent apposition, the healing repair process did not seem to resynthesise elastin proteins (Fig. 3F and Supp. Video 3; the red signal is attributed to red blood cells). However, collagen (green) is mainly present as a fibrotic scar and as the main component of the ECM during reendothelialisation. Furthermore, 3D multiphoton imaging enables researchers to capture the entire layer of endothelial cells and the collagen component of the ECM; DAPI staining is widely used for cell counting. Nevertheless, its broad range of emission signals interferes with the emission signals in the green and red channels, resulting in a yellow signal that is distinguishable from collagen SHG (green) and autofluorescence (red; see Supp. Videos 4-9).

Semi-quantitative data obtained by counting DAPI-positive cells can be very informative when comparing reendothelialisation on different samples (Fig. 4 A-C). Analyses revealed some discrepancy with cell proliferation depending on the samples; $n=11$, $31.47 \% \pm 8.34 \mathrm{SD}$ in (A) in comparison with (B), $n=14 ; 47.9 \% \pm 15$ $\mathrm{SD}$ and (C), $n=11 ; 36,09 \% \pm 9,32 \mathrm{SD}$. The ECM can also be analysed in terms of its directionality by identifying discrepancies between the samples devices (Fig. 4 D-I), i.e., stent in D, G displays collagen fibres that are less organised and less parallel compared to the devices in $\mathrm{E}, \mathrm{H}$.

\section{Discussion}

FDSs are designed to parent vessel reconstruction so as to produce hemodynamic and biological effects that include flow redirection and tissue overgrowth. FDSs provide scaffolding for reendothelialisation and neo-intimal growth [21]. The structure and composition of the FDS materials can affect the healing process, and biophotonic tools such as $3 \mathrm{D}$ in vivo and ex vivo multiphoton imaging are excellent in- 
novative techniques that can implement modern knowledge regarding FDS repair kinetics and biocompatibility.

Fibrillary collagen types I and II are the strongest sources of SHG signals in animal tissues due to their non-centrosymmetric and crystalline natures [22]. Images obtained using multiphoton SHG signals are comparable to conventional histological samples that are visualized with bright field microscopy. The present study demonstrated that collagen fibres in the ECM can be easily evaluated quantitatively, which suggests that collagen ultrastructure as well as novel cells influence reendothelialisation and recovery after a vascular accident. The present information about the 3D stent fibre network will help to further current understandings of reendothelialisation mechanisms after a vascular injury. On the other hand, a slight difference in fibre thickness could not be quantified with SHG signals due to the poor resolution of single collagen fibres [23]. Type III fibres are known to be thinner and produce a much smaller SHG signal than that for type I $[24,25]$, and the properties of the latter may bias the true repartitioning of collagen types in the media and adventitia.

The present study also aimed to use autofluorescence quantification to obtain quantitative results regarding a key ECM protein, elastin, which provides resilience and elasticity to tissues and organs. However, the healing process associated with metallic FDSs (coated or non-coated) did not permit a sufficient amount of elastin to be synthesised in the neo-intima for it to be quantified. The collagen/elastin complex forms an important bearing component in the vascular wall that allows for a properly functioning blood network. Although it is 1000 times more flexible than collagen, elastin operates in partnership with collagen [26] and is the unique regulator of smooth muscle cell proliferation in vivo [27], which suggests that elastin precursors are important candidates for stent biocompatibility. Nevertheless, the repair of damaged elastic fibres in the vascular network remains a challenge for biomedicine [28] because elastic fibre proteins possess extended longevity and are not replaced throughout the lifetime of an organism after their initial developmental synthesis [29]. The present results may shed light on elastin longevity and replacement following vascular accidents and stent apposition.

The present findings demonstrated that ex vivo label-free multiphoton-excited fluorescence can be a valuable tool for the evaluation of aneurysms that have been treated with endovascular devices such as FDS or even coils and for examination of reendothelialisation mechanisms. This technique can be adopted within the scope of multiphoton endoscopy [6,30-33], even in the absence of external dyes and in airways $[34,35]$, to obtain detailed information about the morphology and dynamics of endovascular repair in human tissue. Thus, it may be possible to monitor patients for changes in the endothelium, the therapeutic evolution of the stent in the vicinity of the aneurysm, and changes in the composition of the ECM without requiring interventional biopsies.

\section{Conclusion}

The present study describes novel evaluation modes based on multiphoton microscopy for visualising tissue morphology (e.g., collagen, elastin, and cells) to qualify and quantify the extent of neo-intimal formation of covered arteries and device integration into the arterial wall using a rabbit model of intracranial aneurysms treated with FDS.

\section{Funding}

This research was funded by the French Agence Nationale de la Recherche in the scope of the LabExSigmaLim under the grant number ANR-10-LABX-0074-01.

\section{Disclosure of interest}

The authors declare that they have no competing interest.

\section{Acknowledgements}

The authors thank the French Région Limousin and the French Agence Nationale de la Recherche in the scope of the LabExSigmaLim (ANR-10-LABX-0074-01) for their financial support. The authors are grateful to Y. Launay from the Service commun de Caractérisation des Matériaux de Limoges (CARMALIM) for XPS electron microscopy analyses.

\section{Appendix A. Supplementary data}

Supplementary data associated with this article can be found, in the online version, at https://doi.org/10.1016/j.neurad.2020.03.005.

\section{References}

[1] A. Molyneux, R. Kerr, I. Stratton, et al., International Subarachnoid Aneurysm Trial (ISAT) of neurosurgical clipping versus endovascular coiling in 2143 patients with ruptured intracranial aneurysms: a randomised trial, Lancet 360 (2002) 1267-1274.

[2] I. Arrese, R. Sarabia, R. Pintado, et al., Flow-diverter devices for intracranial aneurysms: systematic review and meta-analysis, Neurosurgery 73 (2013) 193-199, [discussion 99-200].

[3] W. Brinjikji, M.H. Murad, G. Lanzino, et al., Endovascular treatment of intracranial aneurysms with flow diverters: a meta-analysis, Stroke 44 (2013) 442-447.

[4] A.K. Wakhloo, M.J. Gounis, Revolution in aneurysm treatment: flow diversion to cure aneurysms: a paradigm shift, Neurosurgery 61 Suppl 1 (2014) 111-120.

[5] A.Y. Shih, J.D. Driscoll, P.J. Drew, et al., Two-photon microscopy as a tool to study blood flow and neurovascular coupling in the rodent brain, J Cereb Blood Flow Metab 32 (2012) 1277-1309.

[6] G. Ducourthial, P. Leclerc, T. Mansuryan, et al., Development of a real-time flexible multiphoton microendoscope for label-free imaging in a live animal, Sci Rep 5 (2015) 18303.

[7] S.M. Bardet, L. Carr, M. Soueid, et al., Multiphoton imaging reveals that nanosecond pulsed electric fields collapse tumour and normal vascular perfusion in human glioblastoma xenografts, Sci Rep 6 (2016) 34443.

[8] T. Boulesteix, A.M. Pena, N. Pages, et al., Micrometer scale ex vivo multiphoton imaging of unstained arterial wall structure, Cytometry A 69 (2006) 20-26.

[9] A.C. Ko, A. Ridsdale, L.B. Mostaco-Guidolin, et al., Non-linear optical microscopy in decoding arterial diseases, Biophys Rev 4 (2012) 323-334.

[10] H. Miyata, K. Shimizu, H. Koseki, et al., Real-time imaging of an experimenta intracranial aneurysm in rats, Neurol Med Chir (Tokyo) 59 (2019) 19-26.

[11] C. Cavinato, C. Helfenstein-Didier, T. Olivier, et al., Biaxial loading of arterial tissues with 3D in situ observations of adventitia fibrous microstructure: a method coupling multi-photon confocal microscopy and bulge inflation test, J Mech Behav Biomed Mater 74 (2017) 488-498.

[12] X. Yu, R. Turcotte, F. Seta, et al., Micromechanics of elastic lamellae: unravelling the role of structural inhomogeneity in multi-scale arterial mechanics, J R Soc Interface 15 (2018) 20180492.

[13] A. Zoumi, X. Lu, G.S. Kassab, et al., Imaging coronary artery microstructure using second-harmonic and two-photon fluorescence microscopy, Biophys J 87 (2004) 2778-2786.

[14] A.M. Robertson, X. Duan, K.M. Aziz, et al., Diversity in the strength and structure of unruptured cerebral aneurysms, Ann Biomed Eng 43 (2015) 1502-1515.

[15] T.A. Altes, H.J. Cloft, J.G. Short, et al., Creation of saccular aneurysms in the rabbit, AJR Am J Roentgenol 174 (2000) 349-354.

[16] A. Rouchaud, C. Ramana, W. Brinjikji, et al., Wall apposition is a key factor for aneurysm occlusion after flow diversion: a histologic evaluation in 41 rabbits, AJNR Am J Neuroradiol 37 (2016) 2087-2091.

[17] J. Cortese, C. Rasser, G. Even, et al., Optimisation de la biocompatibilité des stents flow-diverters par greffage du P8RI pour le traitement des anévrismes intracrâniens, J Neuroradiol 46 (2019) 67-68.

[18] E.E. Morrill, A.N. Tulepbergenov, C.J. Stender, et al., A validated software application to measure fibre organisation in soft tissue, Biomech Model Mechanobiol 15 (2016) 1467-1478.

[19] D. Plaza-Bonilla, C. Cantero-Martínez, J. Álvaro-Fuentes, et al., Low-cost image analysis method to quantify root surface area (2012).

[20] A.C. Dordea, M.-A. Bray, K. Allen, et al., An open-source computational tool to automatically quantify immunolabeled retinal ganglion cells, Exp Eye Res 147 (2016) 50-56. 
[21] D.F. Kallmes, Y.H. Ding, D. Dai, et al., A new endoluminal, flow-disrupting device for treatment of saccular aneurysms, Stroke 38 (2007) 2346-2352.

[22] X. Chen, O. Nadiarynkh, S. Plotnikov, et al., Second harmonic generation microscopy for quantitative analysis of collagen fibrillar structure, Nat Protoc 7 (2012) 654-669.

[23] J. Mansfield, J. Yu, D. Attenburrow, et al., The elastin network: its relationship with collagen and cells in articular cartilage as visualised by multiphoton microscopy, J Anat 215 (2009) 682-691.

[24] H.A. Eriksen, A. Pajala, J. Leppilahti, et al., Increased content of type III collagen at the rupture site of human Achilles tendon, J Orthop Res 20 (2002) 1352-1357.

[25] M. Suzuki, D. Kayra, W. Elliott, et al., Second harmonic generation microscopy differentiates collagen type I and type III in COPD (2012).

[26] T.C. Gasser, Chapter 8 - Aorta, in: Y. Payan, J. Ohayon (Eds.), Biomechanics of Living Organs, Academic Press, Oxford, 2017.

[27] S.G. Wise, G.C. Yeo, M.A. Hiob, et al., Tropoelastin: a versatile, bioactive assembly module, Acta Biomaterialia 10 (2014) 1532-1541.

[28] M.J. Sherratt, Tissue elasticity and the ageing elastic fibre, Age (Dordr) 31 (2009) 305-325.
[29] S.D. Shapiro, S.K. Endicott, M.A. Province, et al., Marked longevity of human lung parenchymal elastic fibres deduced from prevalence of D-aspartate and nuclear weapons-related radiocarbon, J Clin Invest 87 (1991) 1828-1834.

[30] C.H. Hage, P. Leclerc, M. Fabert, et al., A readily usable two-photon fluorescence lifetime microendoscope, J Biophotonics 12 (2019) e201800276.

[31] A. Lombardini, V. Mytskaniuk, S. Sivankutty, et al., High-resolution multimodal flexible coherent Raman endoscope, Light Sci Appl 7 (2018) 10.

[32] C.M. Brown, D.R. Rivera, I. Pavlova, et al., In vivo imaging of unstained tissues using a compact and flexible multiphoton microendoscope, J Biomed Opt 17 (2012) 040505.

[33] D.M. Huland, C.M. Brown, S.S. Howard, et al., In vivo imaging of unstained tissues using long gradient index lens multiphoton endoscopic systems, Biomed Opt Express 3 (2012) 1077-1085.

[34] S. Kretschmer, M. Pieper, G. Hüttmann, et al., Autofluorescence multiphoton microscopy for visualisation of tissue morphology and cellular dynamics in murine and human airways, Lab Invest 96 (2016) 918-931.

[35] A. Dilipkumar, A. Al-Shemmary, L. Kreiß, et al., Label-free multiphoton endomicroscopy for minimally invasive in vivo imaging, Adv Sci (Weinh) 6 (2019) 1801735-1801835. 